Revue bibliographique pour le domaine irano-aryen

Volume 37-38-39 | 2018

Comptes rendus des publications de 2014-2016

\title{
Chiara Barbati. "The Emergence of the Allographic Phenomenon within the Christian Sogdian Tradition"
}

Florence Jullien

\author{
(2) OpenEdition \\ Journals \\ Édition électronique \\ URL : http://journals.openedition.org/abstractairanica/42950 \\ DOI : 10.4000/abstractairanica.42950 \\ ISBN : 1961-960X \\ ISSN : 1961-960X \\ Éditeur : \\ CNRS (UMR 7528 Mondes iraniens et indiens), Éditions de l'IFRI
}

Référence électronique

Florence Jullien, « Chiara Barbati. "The Emergence of the Allographic Phenomenon within the

Christian Sogdian Tradition" », Abstracta Iranica [En ligne], Volume 37-38-39 | 2018, document 2, mis en ligne le 30 décembre 2018, consulté le 28 septembre 2020. URL : http://journals.openedition.org/ abstractairanica/42950 ; DOI : https://doi.org/10.4000/abstractairanica.42950

Ce document a été généré automatiquement le 28 septembre 2020.

Tous droits réservés 


\title{
Chiara Barbati. "The Emergence of the Allographic Phenomenon within the Christian Sogdian Tradition"
}

\author{
Florence Jullien
}

\section{RÉFÉRENCE}

Chiara Barbati. "The Emergence of the Allographic Phenomenon within the Christian Sogdian Tradition", in J. den Heijer, A.B. Schmidt, T. Pataridze (eds), Scripts beyond Borders. A Survey of Allographic Traditions in the Euro-Mediterranean World, (Publications de l'Institut Orientaliste de Louvain 62), Louvain-la Neuve, 2014, p. 257-278.

1 L'objet de cet article est de montrer comment le type de message véhiculé par un texte sogdien induit des particularités linguistiques et d'écriture. L'A., spécialiste de la tradition sogdienne, présente ici un tableau d'ensemble expliquant les caractéristiques de la langue et de la culture sogdiennes, et leur dette envers le milieu centrasiastique des VIII ${ }^{\mathrm{e}}-\mathrm{XI}{ }^{\mathrm{e}} \mathrm{s}$.

2 Le syriaque, langue liturgique officielle de l'Église d'Orient depuis le $\mathrm{V}^{\mathrm{e}} \mathrm{s}$. et l'écriture syriaque orientale furent les véhicules de la diffusion du christianisme en Asie centrale jusqu'en Chine et vers le sud de l'Inde, avec une expansion particulière sous le patriarcat de Timothée Ier (780-823). Cette écriture fut adoptée pour rédiger les textes en sogdien entre les IX et $\mathrm{X}^{\mathrm{e}} \mathrm{s}$. par la communauté de Bulayïq $(10 \mathrm{~km}$ au nord de Turfān); le syriaque fut relayé par les langues locales comme le sogdien qui joua un rôle fondamental par la suite grâce aux circuits commerciaux marchands.

Née de traductions de textes syriaques originaux (d'où sa syntaxe parfois artificielle), la littérature sogdienne inclut nombre d'emprunts, résultat d'influences de différentes traditions manuscrites centrasiatiques, spécialement syro-orientales et manichéennes. Ce contexte permet de mieux comprendre l'environnement culturel et de développement du phénomène allographique au sein de la tradition chrétienne 
sogdienne. L'A. établit une liste d'exemples de ces interactions mutuelles en soulignant l'importance des traditions littéraires syriaques, manichéennes (dans les langues moyen-iraniennes), mais aussi chrétiennes en pehlevi et en arabe : le support papier par exemple - influence de la tradition manuscrite manichéenne -, l'usage de certaines encres, les en-têtes, l'écriture en colonnes, certaines ornementations dans le texte, l'absence de colophons ou de datation des manuscrits, le recours à des cahiers avec numérotation en lettres syriaques... Un article qui fait commodément le point des connaissances sur le sujet.

\section{AUTEURS}

FLORENCE JULLIEN

CNRS, Mondes iranien et indien, Paris 